\title{
EANM position on the in-house preparation of radiopharmaceuticals
}

\author{
Harry Hendrikse $^{1} \cdot$ Oliver Kiss $^{2} \cdot$ Jolanta Kunikowska ${ }^{3} \cdot$ Wolfgang Wadsak $^{4} \cdot$ Clemens Decristoforo $^{5} \cdot$ Marianne Patt $^{6}$
}

Published online: 20 January 2022

○ The Author(s), under exclusive licence to Springer-Verlag GmbH Germany, part of Springer Nature 2022

\begin{abstract}
The EANM herewith clearly expresses its commitment and support to the non-commercial in-house preparation of radiopharmaceuticals for direct use in accordance with European and national regulations.
\end{abstract}

\section{Introduction}

The daily clinical practice in Nuclear Medicine makes use of radiopharmaceuticals that are either obtained from external commercial suppliers or prepared in-house for immediate use. The latter are usually non-commercial preparations that represent the major source of radiopharmaceuticals for essential routine Nuclear Medicine practices for both diagnostic and therapeutic applications. According to European legislation, namely directive 2001/83/EC, radiopharmaceuticals that are commercially distributed must have a marketing authorization (MA) to be placed on the market. The availability of this type of finished radiopharmaceutical products with MA ready to use is limited due to different reasons:

Clemens Decristoforo and Marianne Patt contributed equally.

This article is part of the Topical Collection on Radiopharmacy.

Wolfgang Wadsak

wolfgang.wadsak@meduniwien.ac.at

1 Department of Clinical Pharmacology \& Pharmacy, Department of Radiology \& Nuclear Medicine, VU University Medical Center, Amsterdam, The Netherlands

2 Department of Radiopharmaceuticals Production, Institute of Radiopharmaceutical Cancer Research, Helmholtz-Zentrum Dresden-Rossendorf, Dresden, Germany

3 Nuclear Medicine Department, Medical University of Warsaw, Warsaw, Poland

4 Department of Biomedical Imaging and Image-guided Therapy, Division of Nuclear Medicine, Medical University of Vienna, AKH Wien, Waehringer Guertel 18-20, A-1090 Vienna, Austria

5 Department of Nuclear Medicine, Medical University Innsbruck, Innsbruck, Austria

6 Department for Nuclear Medicine, University of Leipzig, Leipzig, Germany one is the very short half-life or shelf life, which limits the shipment of these radiopharmaceuticals from external sources. In addition, the market potential for radiopharmaceuticals that are used in rare clinical indications is limited to be financially attractive for pharmaceutical industry, and therefore, the number of MA applications for radiopharmaceuticals is concise.

However, the development of innovative radiopharmaceuticals usually takes place in radiopharmacies, research centers, or nuclear medicine laboratories. Practically all recent major clinical breakthroughs in Nuclear Medicine over the last decade, exemplified by the success of theranostics with Somatostatin analogs and prostate cancer applications, were based on the use of in-house preparations of these innovative products. In case a new radiopharmaceutical has both the technical (half-life) and clinical potential to be produced and distributed commercially, these new radiopharmaceuticals more frequently make their way to pharmaceutical companies that take over from academia and provide funding for further clinical trials besides phase 0/phase I.

European legislation treats radiopharmaceuticals used in the preparation process of a radiopharmaceutical different than other, i.e., non-radioactive pharmaceuticals, by requiring a marketing authorization not only for ready to use radiopharmaceuticals that are to be placed on the market but as well for starting materials such as radionuclide generators, radionuclide precursors, and kits. To avoid misunderstanding, we shall refer throughout the remainder of this document to the term "licensed" for starting materials with a MA.

This document describes the EANM commitment and support to the non-commercial in-house preparation of radiopharmaceuticals for direct use in compliance with European and national regulations, including the "compounding" using licensed starting materials (with MA) such as kits, radionuclide generators, or radionuclide precursors, 
as well as the preparation of diagnostic (PET and SPECT) and therapeutic radiopharmaceuticals using more complex methods and usually unlicensed starting materials (without MA). Starting points for recommendations have been laid down in the guidelines as described in the current Good Radiopharmaceutical Practice (cGRPP) [1].

\section{Specific requirements for the in-house preparation of radiopharmaceuticals}

Like classical (non-radioactive) drugs, the in-house preparation of radiopharmaceuticals must be safe, and the product itself must be effective. However, in-house prepared radiopharmaceuticals have particular fundamental differences, and therefore, pharmaceutical considerations are not always the same as for classical drugs. Major differences compared to classical drugs are as follows:

- Radiopharmaceuticals normally contain only tracer amounts of drug substance, thus lacking a pharmacological effect. This results in a very low toxicological risk profile as compared to classical drugs. The main risk is related to the radioactivity, requiring different considerations.

- The preparation and handling of radiopharmaceuticals need consideration of radiation protection aspects, which require very specific knowledge outside pharmaceutical sciences and therefore highly specialized staff and responsible personnel.

- The small-scale preparation of these radiopharmaceuticals, produced extemporaneously within nuclear medicine or radiopharmacy departments, is mostly for an individual patient or a few patients only. Together with radiation protection considerations, products often have to be prepared in single-dose containers or syringes with a small, insufficient volume to perform extensive testing as applicable for classical drugs.

- There is a much shorter interval between preparation and administration (typically within minutes to hours) compared to classical drugs due to their very short halflives. This immediate application together with typical formulations without growth-promoting properties and small volumes applied reduces the risks related to the microbial contamination level for intravenously administered radiopharmaceuticals significantly.

- Radiopharmaceuticals are released to nuclear medicine specialists with respective training and expertise, but never to patients themselves. Administration of the radiopharmaceutical is always done in a controlled environment typically within a hospital and patients remain under supervision until the investigation or treatment is finished.

- Radiopharmaceuticals in most circumstances cannot be fully tested before release (e.g., sterility testing) or stored over longer periods of time due to radioactive decay.

- In the case of the use of licensed starting materials only, the risk for the preparation of defective radiopharmaceuticals is almost excluded and covered by the marketing authorization holder of the starting materials.

To accommodate these differences, EANM has released dedicated guidelines and guidance documents for this well-established practice. The main guidance for the small-scale preparation of radiopharmaceuticals has been published in the EANM cGRPP guidelines [1]. It follows the structure of the EU Good Manufacturing Practice (GMP) guidelines [2] and covers both the practice of preparations from licensed kits and generators, as well as those using more complex procedures in separate chapters. It describes specific considerations regarding the facilities, taking into account the specific environment found in hospitals and other healthcare establishments related to facilities and clean room grading. It clearly defines responsibilities and the needs for specific training in this practice, as well as clear guidance for aseptic procedures, quality control documentation, and many other specific aspects.

Supporting this general document, specific EANM guidelines related to cGRPP have been published on automated systems [3], process and method validation [4, 5], or are in preparation (EANM Guideline on "Quality Risk Management guidelines applied to radiopharmaceuticals," 2021). EANM congresses and EANM educational programs complement these guidelines.

Additionally, since more than 20 years, EANM provides a European post-graduate specialization certificate in Radiopharmacy, based on dedicated post-graduate courses specifically designed for the small-scale preparation of radiopharmaceuticals. This certificate is available to anyone who completes the defined program of education and has 2 years of practical experience in the field, and EANM considers this to be the basis for being responsible for the in-house preparation of radiopharmaceuticals (see: https:// www.eanm.org/esmit/radiopharmacy-certification/). Specific educational EANM activities within the European School of Multimodality Imaging \& Therapy (ESMIT) ensure continuing educational programs for professionals in the field, taking into account the rapid developments in the field. 
Table 1 Overview of legally binding and guidance documents for radiopharmaceuticals in Europe (adapted from [7, 8]

\begin{tabular}{|c|c|c|c|}
\hline & \multicolumn{3}{|l|}{ Categories of radiopharmaceuticals } \\
\hline & Marketing authorization & Clinical trials & In-house preparations \\
\hline \multirow[t]{6}{*}{ Legally binding documents } & \multicolumn{3}{|c|}{ Ph. Eur. General and Specific Monographs } \\
\hline & Directive 2001/83/EC & Directive 2001/20/EC & National governance \\
\hline & Directive 2003/94/EC & Directive 2003/94/EC & \\
\hline & Directive 2004/27/EC & Directive 2005/28/EC & \\
\hline & GMP Annex 3 & Regulation 536/2014 & \\
\hline & & GMP Annex 13 & \\
\hline \multirow[t]{4}{*}{ Guidance documents } & \multirow{4}{*}{$\begin{array}{l}\text { EMA Guideline on Radiopharma- } \\
\text { ceuticals }\end{array}$} & EC Guidance IMP/NIMP & Ph. Eur. General Chapter 5.19 \\
\hline & & EMA Guideline IMPD & PIC/S GPP 010-4 incl. Annex 3 \\
\hline & & $\begin{array}{l}\text { EMA Guideline first-in-human clini- } \\
\text { cal trials }\end{array}$ & $\begin{array}{l}\text { EANM guidelines and guidance } \\
\text { documents }\end{array}$ \\
\hline & & $\begin{array}{l}\text { EANM guidelines and guidance } \\
\text { documents }\end{array}$ & National documents \\
\hline
\end{tabular}

\section{Current regulation of non-commercial in-house preparation of radiopharmaceuticals}

Radiopharmaceuticals have to meet requirements according to two sources of legislation, namely pharmaceutical legislation and radiation protection regulations. The role of radiation protection legislation is out of the scope of this position paper. Non-commercial in-house preparation is mostly performed in nuclear medicine departments, research centers, and other radiopharmaceutical laboratories. Applications of non-commercially in-house prepared radiopharmaceuticals comprise both clinical routine procedures (diagnostic or therapeutic) as well as clinical trials and basic research.

The in-house preparations can be divided into two major categories:

- Radiopharmaceuticals prepared from licensed kits, generators, and/or radionuclide precursors.

- Radiopharmaceuticals prepared from unlicensed starting materials (either manual synthesis or automated procedure using synthesis modules).

Most countries in Europe treat these two categories slightly different with regard to applicable GMP requirements, thus acknowledging the low risk associated with preparations that are made exclusively from licensed starting materials in accordance with the instructions provided by the MA holder.

However, whereas clear regulations are defined in directive 2001/83/EC for ready to use radiopharmaceuticals that are distributed commercially, varying national regulatory interpretations for in-house produced radiopharmaceuticals are in place throughout Europe. Considering that radiopharmaceutical in-house preparation resembles magistral prescriptions/extemporaneous preparations of drugs within pharmacies rather than industrial manufacturing, this also implies a different regulatory framework compared to industrial procedures related to authorization of the facility, quality assurance (GMP) requirements, and responsible personnel. However, the quality of the final radiopharmaceuticals and their control is defined in the same way, based on the European Pharmacopeia providing general information, namely the general monograph 0125 , and respective specific monographs [6].

It is noteworthy to state that current European pharmaceutical legislation does not enforce a preferred use of a commercially available ready to use radiopharmaceutical with MA over an in-house produced product.

For the preparation of in-house radiopharmaceuticals, the underlying regulatory framework for preparing these radiolabeled drugs can be roughly divided in legally binding documents (mandatory) and guidance documents (recommendations). An overview is given in Table 1 (from [7, 8]). For in-house production of radiopharmaceuticals, specific guidance documents are available, including Ph. Eur. General Chapter on extemporaneous preparation of radiopharmaceuticals [9], PIC/S GPP 010-4 (incl. Annex 3) [10], dedicated EANM Guidelines ([1, 3-5], and national guidance documents.

The Clinical Trial Regulation (Regulation (EU) No $536 / 2014$ ), valid from 2022, provides the basis to apply the same considerations for in-house produced IMPs as for clinical routine applications with respect to the facility and GMP requirements [11]. Additional guidance for IMPs can be found in GMP Annex 13; EC Guidance IMP/ NIMP; EMA Guideline IMPD; and EMA Guideline firstin-human clinical trials [12]. 


\section{Summary and conclusions}

This paper tries to describe the specifics of in-house preparation of radiopharmaceuticals, which today is an essential practice for Nuclear Medicine. The need for in-house preparations will even increase in the future taking into account the rapid development of the field, both in terms of technical advances (e.g., new radionuclides, new technologies for automated production) and also in utilizing new clinical relevant targets in many fields and further developing personalized medicine approaches, e.g., in the context of theranostics. To ensure appropriate standards, EANM maintains numerous activities related to guidelines and education that guarantee safety and efficacy of Nuclear Medicine applications and to facilitate patient access to innovative and highly effective diagnostic and therapeutic approaches. The current regulatory framework for in-house preparation, unfortunately, is not harmonized throughout Europe and has resulted in unbalanced access to innovative radiopharmaceuticals, based on national particularities [12]. Future changes in the European legislation should consider the importance of in-house preparation of radiopharmaceuticals, ensuring quality and safety with harmonized standards and dedicated rules taking into account the particular needs for this practice, simply for the benefit of patients otherwise having delayed or even no access to often life-saving treatments.

\section{Declarations}

Without direct relevance to this work, WW declares that he is a part time employee of $\mathrm{CBmed} \mathrm{GmbH}$, Graz, Austria, and a co-owner of MINUTE medical GmbH, Vienna, Austria. Without direct relevance to this work, JK reports consulting fees from Telix; and participation on a Data Safety Monitoring Board and Advisory Board from Novartis (personal fees). All other authors have no relevant financial or nonfinancial interests to disclose.

Ethics approval Institutional Review Board approval was not required because the manuscript is an Editorial.

Consent to participate Not applicable.

\section{References}

1. Gillings N, Hjelstuen O, Ballinger J, Behe M, Decristoforo C, Elsinga $\mathrm{P}$, et al. Guideline on current good radiopharmacy practice
(cGRPP) for the small-scale preparation of radiopharmaceuticals. EJNMMI radiopharm chem. 2021;6(1):8.

2. Daue R. EudraLex - Volume 4 - Good Manufacturing Practice (GMP) guidelines [Internet]. Public Health - European Commission. 2017. https://ec.europa.eu/health/documents/eudralex/vol-4 en. Accessed 7 Jan 2022.

3. Aerts J, Ballinger JR, Behe M, Decristoforo C, Elsinga PH, Faivre-Chauvet A, et al. Guidance on current good radiopharmacy practice for the small-scale preparation of radiopharmaceuticals using automated modules: a European perspective. J Labelled Comp Radiopharm. 2014;57(10):615-20.

4. Todde S, Peitl PK, Elsinga P, Koziorowski J, Ferrari V, Ocak EM, et al. Guidance on validation and qualification of processes and operations involving radiopharmaceuticals. EJNMMI radiopharm chem. 2017;2(1):8.

5. Gillings N, Todde S, Behe M, Decristoforo C, Elsinga P, Ferrari V, et al. EANM guideline on the validation of analytical methods for radiopharmaceuticals. EJNMMI Radiopharm Chem. 2020;5(1):7.

6. Council of Europe, European Pharmacopoeia Commission, European Directorate for the Quality of Medicines \& Healthcare (2020). European pharmacopoeia 10th edition

7. Lange R, ter Heine R, Decristoforo C, Peñuelas I, Elsinga PH, van der Westerlaken MML, et al. Untangling the web of European regulations for the preparation of unlicensed radiopharmaceuticals: a concise overview and practical guidance for a risk-based approach. Nucl Med Commun. 2015;36(5):414-22.

8. Decristoforo C, Neels O, Patt M. Emerging radionuclides in a regulatory framework for medicinal products - how do they fit? Front Med. 2021;28(8):678452.

9. 5.19. Extemporaneous preparation of radiopharmaceuticals. In: European Pharmacopoeia. 10.0. European Directorate for the Quality of Medicines \& HealthCare (EDQM); p. 807-12.

10. PIC/S guide to good practices for the preparation of medicinal products in healthcare establishments. Available from: https:// picscheme.org/docview/3443. Accessed 9 Feb 2021

11. Peñuelas I, Vugts DJ, Decristoforo C, Elsinga PH. The new regulation on clinical trials in relation to radiopharmaceuticals: when and how will it be implemented? EJNMMI Radiopharm Chem. 2019;4(1):2.

12. Decristoforo C, Penuelas I, Patt M, Todde S. European regulations for the introduction of novel radiopharmaceuticals in the clinical setting. Q J Nucl Med Mol Imaging. 2017;61(2):135-44.

Publisher's note Springer Nature remains neutral with regard to jurisdictional claims in published maps and institutional affiliations. 